\title{
Portable Wireless Device Threat Assessment for Aircraft Navigation Radios
}

\author{
Truong X. Nguyen, Sandra V. Koppen, Jay J. Ely, \\ Reuben A. Williams, Laura J. Smith \\ NASA Langley Research Center \\ Hampton, Virginia
}

\author{
Maria Theresa P. Salud \\ Lockheed Martin \\ Hampton, Virginia
}

\begin{abstract}
This paper addresses the concern for Wireless Local Area Network devices and two-way radios to cause electromagnetic interference to aircraft navigation radio systems. Spurious radiated emissions from various IEEE 802.11a, 802.11b, and Bluetooth devices are characterized using reverberation chambers. The results are compared with baseline emissions from standard laptop computer and personal digital assistants (PDAs) that are currently allowed for use on aircraft. The results indicate that the WLAN devices tested are not more of a threat to aircraft navigation radios than standard laptop computers and PDAs in most aircraft bands. In addition, spurious radiated emission data from seven pairs of two-way radios are provided. These twoway radios emit at much higher levels in the bands considered. A description of the measurement process, device modes of operation and the measurement results are reported.
\end{abstract}

Keywords-WLAN, FRS, GMRS spurious emission, aircraft, communication, navigation, interference, 802.11, Bluetooth, EMI, reverberation.

\section{INTRODUCTION}

Wireless technologies are widely adopted in the present consumer market. Technologies such as cellular phones and Wireless Local Area Networks (WLANs) have brought a revolution in accessibility and productivity. WLANs enable consumers to have convenient access to web-browsing, email, instant messaging and numerous enterprise applications. As travelers become more dependent upon Internet access, airlines are increasingly interested in providing connectivity to their customers while traveling onboard aircraft. While WLAN equipment provided by the airlines for permanent installation on the aircraft must be properly certified, passenger carry-on products are not required to pass the rigorous aircraft radiated field emission standards.

Two-way radio communications, such as Family Radio Service (FRS) and General Mobile Radio Service (GMRS), are also becoming popular. These no-fee radio systems allow family members, friends and business associates to stay in contact during trips, shopping, or where party members may be physically dispersed. Unlike the low power FRS radios with half-watt maximum transmitted power, GMRS radio can radiate much higher power. Two-watt GMRS radio

This work was funded by the Federal Aviation Administration and the NASA Aviation Safety Program as part of the Interagency Agreement DFTA03-96-X-9000, Revision 9. models, which require a license presently, are highly popular. Many recent models have both FRS and GMRS built-in features. While use of these radios is not presently authorized on aircraft, their low cost and popularity hint that their use by unsuspecting passengers is likely.

This paper documents the spurious radiated emission measurement process and the results from WLAN devices and two-way radio testing in the aircraft navigation radio bands. The emission results are compared against emissions from standard laptop computers and Personal Digital Assistants, which are used in this paper as benchmarks since the use of these devices are currently allowed during certain non-critical phases of flight.

Data in this paper come from a larger effort to assess interference of wireless devices to aircraft systems. In this larger effort, device spurious emissions, aircraft interference path loss, radio receiver susceptibility thresholds, and interference risk assessments were considered [1]. Only wireless device emission measurement results are shown in this paper.

\section{APPROACH}

Radiated emission measurements were performed in a reverberation chamber for optimal speed and repeatability. The results are provided directly in terms of effective peak radiated power.

Wireless LAN devices tested include seven IEEE $802.11 \mathrm{~b}$, five IEEE 802.11a, and six Bluetooth devices (Bluetooth is actually classified under Wireless Personal Area Network, WPAN, but is grouped under WLAN label in this report for simplicity). Also measured were emissions from seven pairs of two-way radios. Fig. 1 and 2 show the devices considered in this study.
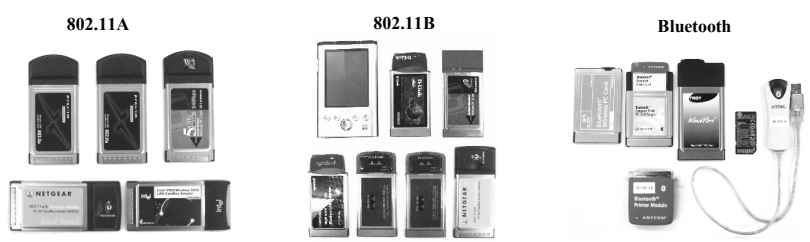

Figure 1. IEEE 802.11a, 802.11b and Bluetooth Devices. 


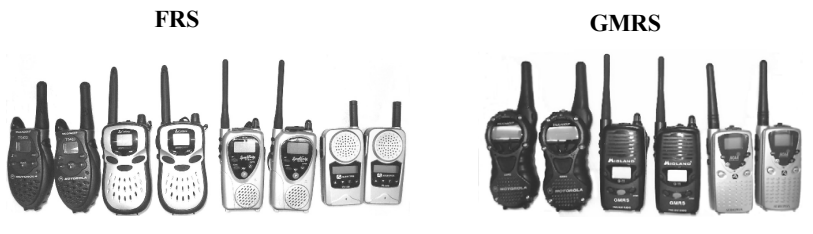

Figure 2. FRS and GMRS Two-Way Radios.

Table I summarizes capabilities and frequencies associated with each technology.

Computers with built-in WLAN capability were not common at the time this study was conducted. Most WLAN devices were in form of plug-in or external that required a separate host computer or PDA. As a result, preliminary testing was conducted for selecting host laptop computers and PDAs with low emissions so that their emissions would not mask the emissions from the WLAN devices. The screening involved measurement of eight laptop computers and two PDAs in various operational modes. The host laptops and PDAs were selected using the criteria of the lowest emissions while operating in idle and file transfer modes since they were typical laptop modes during tests. This screening identified two laptop computers and both PDAs to cover five measurement frequency bands.

Extensive measurements were also conducted on the same eight laptop computers and two PDAs to establish emission baselines of devices currently allowed for use on aircraft. During this baseline measurement, the devices were set to various operating modes, including $\mathrm{CD}$ and DVD playing, file transfer, idle, and screensaver modes. The comparisons between emissions from the WLAN devices and the baseline laptops/PDA are shown and compared against emissions limits from the FCC Part 15 and the RTCA-DO160D Category $\mathrm{M}$ for cabin equipment.

Several aircraft radio bands were considered, including Localizer (LOC), Glideslope (GS), Very High Frequency Omnidirectional Range (VOR), Global Positioning Systems (GPS), Traffic Collision Avoidance System (TCAS), Distance Measuring Equipment (DME), and Microwave Landing Systems (MLS).

To reduce the number of emission measurements, aircraft radio bands that overlapped, or were near one another were grouped together, and emissions were measured across the entire combined band simultaneously. Five frequency

TABLE I. WIRELESS AND RADIO TECHNOLOGY SUMMARY

\begin{tabular}{|c|c|c|c|c|}
\hline & $\begin{array}{c}\text { Approx } \\
\text { Freq. } \\
\text { Range }\end{array}$ & $\begin{array}{c}\text { Typical } \\
\text { Data Rates } \\
(\text { Mbps })\end{array}$ & $\begin{array}{c}\text { Max Power } \\
(\mathbf{m W}) \text { per } \\
\text { Standard/Test }\end{array}$ & $\begin{array}{c}\text { Range } \\
(\mathbf{m})\end{array}$ \\
\hline $802.11 \mathrm{a}$ & $5.15-5.825$ & $6,12,24,54$ & $800 / 40 \& 200$ & 50 \\
\hline $802.11 \mathrm{~b}$ & $2.4-2.4835$ & $1,2,5.5,11$ & $1000 / 100$ & $24-100$ \\
\hline Bluetooth & $2.4-2.4835$ & 1 & $100 /<1$ & $10-100$ \\
\hline FRS & $0.462-0.468$ & NA & $500 / 500$ & 2 miles \\
\hline GMRS & $0.462-0.468$ & NA & $5000 / 2000$ & 5 miles \\
\hline
\end{tabular}

groups, designated as measurement Band 1 to Band 5, covered all aircraft radio bands of interest. Table II correlates the measurement bands to aircraft radio frequencies.

It is assumed in this study that high emissions in any of Bands 1 through 5 may affect all aircraft systems operating in that band. As an example, high emissions in Band 1 are assumed to affect all LOC and VOR systems as a group. No effort was taken to distinguish whether the emissions were in LOC or VOR bands.

Various filter combinations were used in the antenna path of the Access Point (AP) to allow only the intended wireless signal for communicating with the Devices-Under-Test (DUTs), while blocking spurious emissions from the APs. Additional filters were also used in the measurement path to prevent the wireless signals (from the wireless cards and the AP) from reaching the measuring instrument to cause overloading or intermodulation.

Measurements were conducted on four matched pairs of FRS radios and three matched pairs of GMRS radios. Each of these devices can operate independently with out a host.

Emissions from a matched pair were measured at the same time, with each radio in turn being in transmit, receive, and idle modes. Thus, a recorded measurement trace includes the maximum emissions from both radios in all three modes. The radios were also cycled through at least two frequency channels during each measurement. Again, filters were employed to prevent overloading of the measurement receiver.

\section{MEASUREMENT MethoD}

The reverberation chamber method used is a relatively new method that compares the test chamber's power density statistics from an injected calibration signal against the same statistics resulting from unknown signals radiated by the test device. A pair of matched, in-band antennas is typically used to inject the calibration signal and to receive (or pickup) RF signal from the chamber. A set of continuous rotating conducting paddles, called stirrers, is used to change the boundary conditions in the chamber while the receive antenna samples chamber power density for deriving field

TABLE II. EMISSION MEASUREMENT BANDS AND CORRESPONDING AIRCRAFT RADIO BANDS

\begin{tabular}{|c|c|c|c|}
\hline $\begin{array}{c}\text { Measure- } \\
\text { ment } \\
\text { Band }\end{array}$ & $\begin{array}{c}\text { Measurement } \\
\text { Freq. Range } \\
\text { (MHz) }\end{array}$ & $\begin{array}{c}\text { Aircraft } \\
\text { Systems } \\
\text { Covered }\end{array}$ & $\begin{array}{c}\text { Spectrum } \\
\text { (MHz) }\end{array}$ \\
\hline \multirow{2}{*}{ Band 1 } & $105-120$ & LOC & $108.1-111.95$ \\
\cline { 3 - 4 } Band 2 & $325-340$ & VOR & $108-117.95$ \\
\hline \multirow{2}{*}{ Band 3 } & \multirow{2}{*}{$960-1250$} & GS & $328.6-335.4$ \\
\cline { 3 - 4 } & & TCAS & 1090 \\
\cline { 3 - 4 } & & ATCRBS & 1030 \\
\cline { 3 - 4 } & & GPS L2 & 1227.60 \\
\hline Band 4 & $1565-1585$ & GPS L5 & 1176.45 \\
\hline Band 5 & $5020-5100$ & GPS L1 & $1575.42 \pm 2$ \\
\hline
\end{tabular}


statistics. This helps eliminate the need to move the receive antenna around for the sampling.

The signal statistics typically include the peak receivepower or the average receive-power over a paddle rotation. For emission measurement, peak received power measurement is much faster, and is more appropriate for this application since spurious signals often do not exist long enough to be averaged over a paddle revolution.

\section{A. Calibration}

Test chamber calibration was first performed to determine the composite gain that was then used to correct the measured received power data to arrive at the final emission results. The composite gain includes effects of chamber loss, antenna losses, cable losses, pre-amplifier gain and other losses that may exist along the RF propagation path. During calibration, an in-band antenna was used to transmit a known swept continuous-wave (CW) signal into the chamber, while the spectrum analyzer measured the maximum (peak) signal coupling into an in-band receive antenna. The calibration signal and the spectrum analyzer were frequency tracked. The difference, in $\mathrm{dB}$, between the peak received signal (after a stirrer revolution) and the calibration transmitted power resulted in the chamber's "composite gain". This gain is the calibration factor to be applied to DUT's measured emission data to arrive at DUT's emission level. Chamber field uniformity was demonstrated in an earlier effort, and is not repeated in this study.

Typically a two-step chamber calibration process is used. In the two-step process, field uniformity is first demonstrated for an empty chamber and empty chamber calibration factor is measured. Then, a chamber loading factor (CLF) is measured when test objects are introduced. If this loading factor exceeds $3 \mathrm{~dB}$, it is used to correct the empty chamber calibration factor.

In this effort, a simplified one-step calibration process was used since chamber field uniformity has been demonstrated in a earlier effort. This one-step process requires that the test devices and operator be present in the chamber during calibration. The typical chamber loading factor measurement is no longer needed, eliminating uncertainties about whether a correction for CLF should be applied. To reduce the burden on the operator, calibration was measured at only one receive antenna location rather multiple locations. The effect is a small increase in uncertainty (of less than two $\mathrm{dB}$ depending on chamber field uniformity and frequency). This is considered acceptable for this application.

The faster mode-stirred approach is used, where the stirrers are continuously rotated, rather than the mode-tuned approach with the stirrers slowly stepped. The measurement instrument (spectrum analyzer) used is fast enough for emission measurement purposes. In addition, calibration and measurement times were set the same to reduce the uncertainty associated with non-equal number of points. At a large number of measurements, this uncertainty is expected to be small, however. A typical measurement is two minutes long, with five second per stirrer revolution, and a spectrum analyzer sweep time of between $50 \mathrm{msec}$ to $800 \mathrm{msec}$ depending on frequency bands.

It is often convenient to include the transmit and receive path losses in the chamber calibration measurements. These path losses account for the presence of test cables, in-line amplifiers, attenuators and filters for various purposes. Transmit path losses are associated with components connecting the source output and the transmit antenna, whereas receive path losses are associated with components connecting the receive antenna and the spectrum analyzer input.

As shown in Fig. 3, measurement instrumentation included a spectrum analyzer, a tracking source (frequencycoupled with the spectrum analyzer), a computer, a stirrer controller, transmit and receive antennas, RF filters, preamplifiers, and an IEEE-488 bus. The transmit and receive antennas include dual-ridge horns for frequencies above 960 $\mathrm{MHz}$ and log-periodic antennas for frequencies below. In addition, a GPS survey antenna with built-in filter and amplifier was used as the receive antenna in the GPS band.

\section{B. Emission Measurement and Set Up}

After calibration was completed, the test units were introduced and exercised through various operating modes while their emissions were being measured. The spectrum analyzer was put in maximum trace hold mode while continuously sweeping over the measurement frequency band. The transmit path was capped with a 50-ohm termination to prevent leakage from the tracking source into the test chamber. The composite gain, and the transmit cable loss, was then applied to the measurement results to yield total radiated powers (within measurement resolution bandwidth). Reference [1] provides additional information on the method.

Fig. 3 shows the position of a host and WLAN device in the center of the chamber, represented by a laptop computer on a foam block test stand. The two stirrers located in the corners of the chamber were continuously rotated at $5 \mathrm{rpm}$ during chamber calibrations and emissions testing.

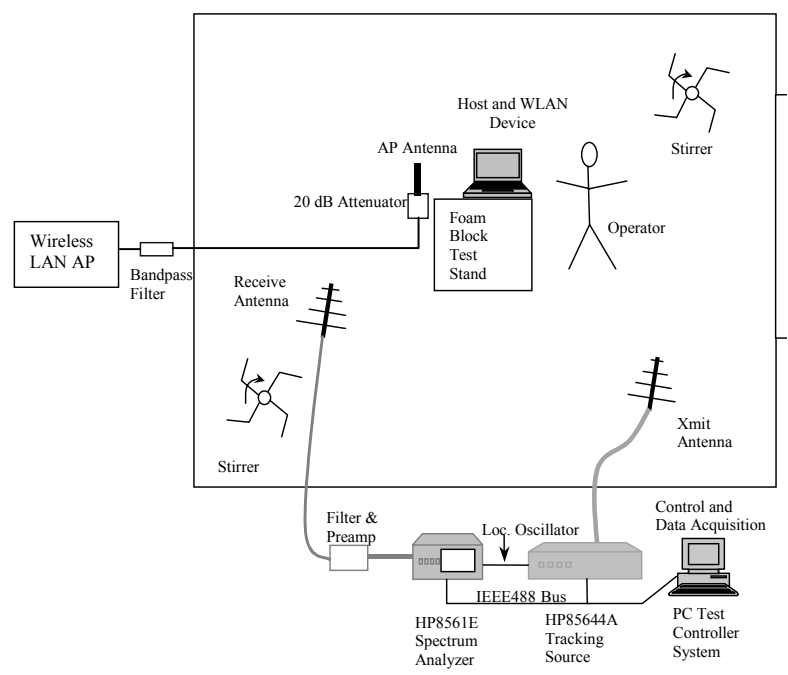

Figure 3. Reverberation Chamber and Set Up. 
RF filters and a preamplifier are indicated in the receive path. The wireless network is illustrated outside the chamber, and includes an AP, a router, a wired laptop, and a bandpass filter inline with the AP antenna. The AP antenna and the wireless card were placed close to each other to overcome multipath interference, and as a result a $20 \mathrm{~dB}$ attenuator was used to reduce signal overload. The AP antenna port that was not used was capped with a 50-ohm termination to prevent signals outside the chamber from coupling in through the AP.

\section{Measurement Result}

The results from the radiated emission tests include emission data from WLAN devices (combined with a host), based on 802.11a, 802.11b, and Bluetooth wireless standards, and FRS and GMRS radios. The results were produced with different combinations of measurement bands and wireless/radio technologies. However, only a representative set of data for GPS band are shown here due to space limitation. The remaining results can be found in [1]. In addition, emission results from standard laptop computers and PDA measuring using the same process, facility and instrument are shown for comparison.

Each trace in each figure is the emission envelope for an individual device in a measurement band. The individual device emission envelopes were generated from measured emission data that included all test modes. For WLAN devices, these test modes include Ping Storm, Duplex File Transfer, and idle mode tests. In the Ping Storm and File Transfer mode, four data rates were tested, along with three WLAN frequency channels for each data rate. Thus each WLAN trace in a band represent approximately 27 different measurements for 802.11a devices and 21 measurements for $802.11 \mathrm{~b}$ devices. About four percent of all measurements failed to yield data due to difficulties maintaining wireless connectivity in the highly reverberating environment. Bluetooth devices were tested only in idle and paging modes.

For laptop computers, each trace represent five measurements with each computer operating in idle, file transfer, CD playing, DVD playing, and graphic-intensive screen saver mode (to exercise the computing engine). For PDA, each trace represents idle and file transfer modes.

For FRS and GMRS radios, the test modes include idle and two to three frequency channels, with the devices in receive and transmit modes.

\section{A. Wireless Devices Results}

Results for WLAN devices and two-way radios are shown in Fig. 4 to 8 for GPS band.

\section{B. Laptops/PDAs Results}

Measurements of laptop computers and PDAs are shown in Fig. 9.

\section{Summary of Results}

Table III summarizes the measurement results. The data shown are the peak emissions of all measurements in a measurement band for a class of wireless device. These

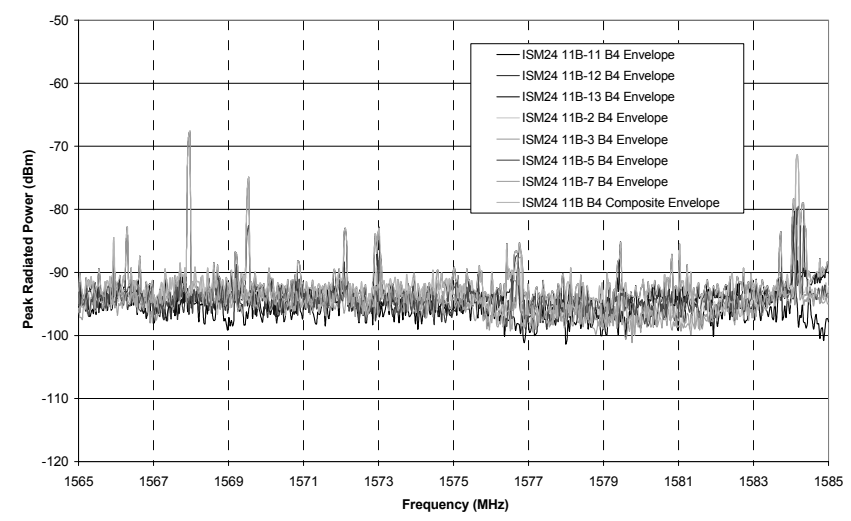

Figure 4. 802.11b Device Radio Emission in GPS Band.

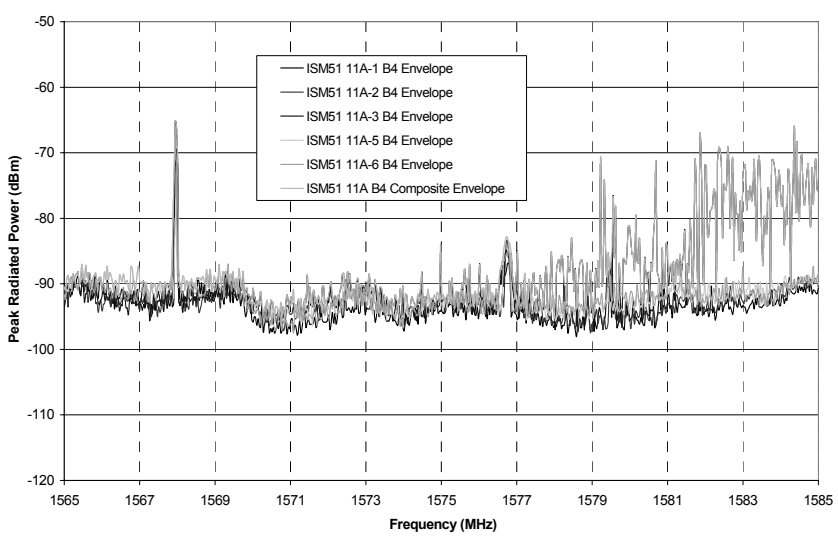

Figure 5. IEEE 802.11a Devices Radio Emission in GPS Band.

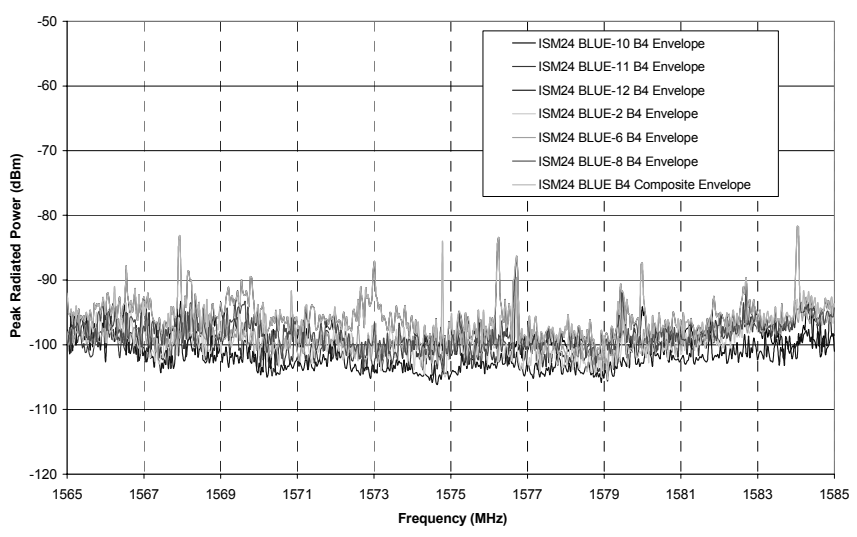

Figure 6. Bluetooth Devices Radio Emission in GPS Band.

data are graphically presented in a later section for comparison with FCC and aircraft equipment emission limits.

\section{COMPARISON WITH FCC AND RTCA/DO-160D LIMITS}

The emission results are in Total Radiated Power (TRP) within the measurement bandwidth. They are compared against the corresponding FCC and aircraft equipment RTCA/DO-160 Category M [2] emission limits. FCC Part 


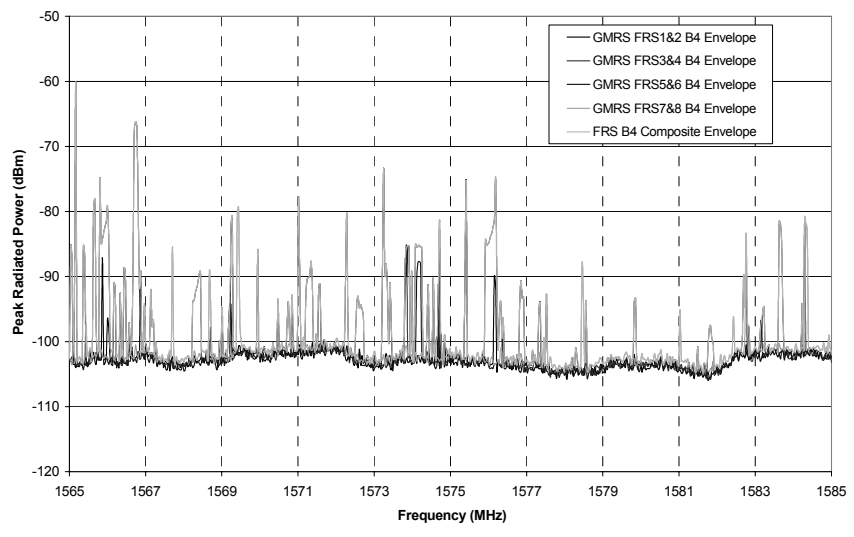

Figure 7. FRS Radio Emission in GPS Band.

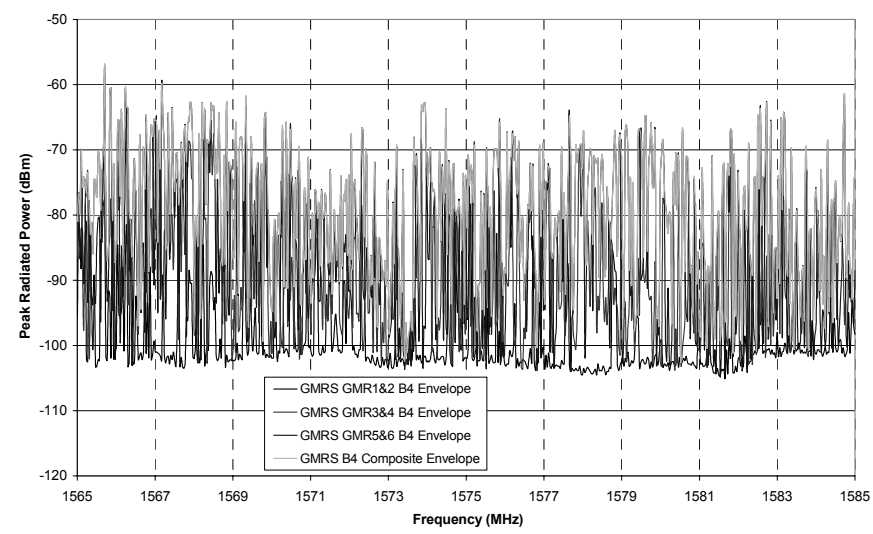

Figure 8. GMRS Radio Emission in GPS Band.

15.109 [3] and 15.209 [4] provide limits for unintentional and intentional radiator. RTCA/DO-160 Category $\mathrm{M}$ is suitable for equipment and associated interconnecting wiring located in the passenger cabin or in the cockpit of a transport aircraft. These limits are given in field strength measured at a given distance from the device, typically $1 \mathrm{~m}$ or $3 \mathrm{~m}$. For comparison with the measured data, the limits are converted to equivalent Effective Isotropic Rated Power (EIRP) using:

$$
E I R P=\left(E^{2} \cdot 4 \pi R^{2}\right) / 377
$$

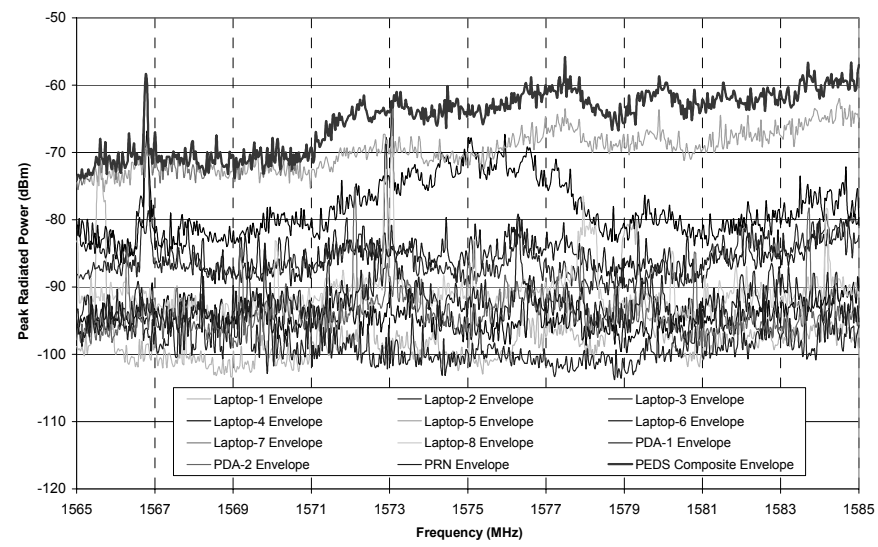

Figure 9. Laptops/PDAs Radio Emission in GPS Band.
TABLE III. MAXIMUM MEASURED EMISSIONS IN AIRCRAFT BANDS

\begin{tabular}{|c|c|c|c|c|c|c|c|}
\hline $\begin{array}{c}\text { Meas. } \\
\text { Band }\end{array}$ & $\mathbf{8 0 2 . 1 1 b}$ & $\begin{array}{c}\text { Blue- } \\
\text { tooth }\end{array}$ & $\mathbf{8 0 2 . 1 1 a}$ & FRS & GMRS & $\begin{array}{c}\text { Laptops } \\
\text { PDAs }\end{array}$ & $\begin{array}{c}\text { Aircraft } \\
\text { Bands }\end{array}$ \\
\hline Band 1 & -78.2 & -66.8 & -74.2 & -90.7 & -79.3 & -68.0 & LOC, VOR \\
\hline Band 2 & -75.7 & -77.2 & -71.8 & -37.2 & -28.5 & -58.7 & GS \\
\hline Band 3 & -65.3 & -49.7 & -57.7 & -43.5 & -44.7 & -45.7 & $\begin{array}{c}\text { TCAS, ATC, } \\
\text { GPS L2,L5 }\end{array}$ \\
\hline Band 4 & -67.7 & -81.7 & -65.2 & -60.2 & -57.0 & -55.8 & GPS L1 \\
\hline Band 5 & -77.7 & -78.2 & -52.0 & -38.2 & -33.0 & -77.0 & MLS \\
\hline
\end{tabular}

$E(\mathrm{~V} / \mathrm{m})$ is electric field strength measured at distance $R$ $(m)$, in the direction of maximum radiation. To convert power, EIRP, from watts to $\mathrm{dBm}$, the expression $10 *$ $\log \left(1000^{*} E I R P\right)$ is used. Table IV shows the maximum FCC and RTCA/DO-160D limits in the measurement bands along with their equivalent EIRP limits.

For simplicity, this paper assumes unity DUT's directivity, thus EIRP was used in the comparison between measured radiated power and FCC and RTCA emission limits. Using EIRP tends to over-estimate the actual radiated power by an amount equivalent to the device's directivity. Reference [5] provides a method to estimate the expected directivity for non-intentional radiators. For computer laptop size, the estimation results in $5 \mathrm{~dB}$ near $100 \mathrm{MHz}$ to about 8 $\mathrm{dB}$ near $5 \mathrm{GHz}$. This estimation of directivity is yet to be widely accepted, and are therefore not used in this paper.

FCC 95.635 [6] provides Effective Radiated Power $(E R P)$ emission limits for FRS and GMRS radio at $-13 \mathrm{dBm}$ outside of the intended transmission frequency. Since the limit is specified as power level, a field-to-power conversion is not needed. In addition, ERP and EIRP are related as:

$$
E R P=E I R P-2.15
$$

Fig. 10 and 11 show the results comparison for the WLAN devices, laptop computers and PDA, and FCC and RTCA/DO-160 limits.

\section{FINDINGS}

1. The results indicate that in most aircraft bands under consideration, the maximum emissions from the WLAN devices tested did not exceed the maximum

\begin{tabular}{|c|c|c|c|c|c|}
\hline & $\begin{array}{c}\text { FCC } \\
\text { Part 15 } \\
(\mu \mathrm{V} / \mathrm{m} \\
\text { a } 3 \mathrm{~m})\end{array}$ & $\begin{array}{c}\text { DO-160 } \\
\text { Cat. M } \\
(\mathrm{dB} \mu \mathrm{V} / \mathrm{m} \\
\text { a } 1 \mathrm{~m})\end{array}$ & $\begin{array}{c}\text { Equiv. } \\
\text { FCC } \\
\text { Part } 15 \\
(E I R P, \\
\text { dBm) }\end{array}$ & $\begin{array}{c}\text { Equiv. } \\
\text { DO-160 } \\
\text { Cat. M } \\
(E I R P, \\
\text { dBm })\end{array}$ & $\begin{array}{c}\text { FCC FRS/ } \\
\text { GMRS } \\
(E R P, \\
\text { dBm) }\end{array}$ \\
\hline Band 1 & 150 & 34 & -51.7 & -70.8 & -13 \\
\hline Band 2 & 200 & 52.9 & -49.2 & -51.9 & -13 \\
\hline Band 3 & 500 & 50 & -41.2 & -54.8 & -13 \\
\hline Band 4 & 500 & 53 & -41.2 & -51.8 & -13 \\
\hline Band 5 & 500 & 71.8 & -41.2 & -33.0 & -13 \\
\hline
\end{tabular}

TABLE IV. FCC AND RTCA LIMITS IN AIRCRAFT RADIO BANDS 


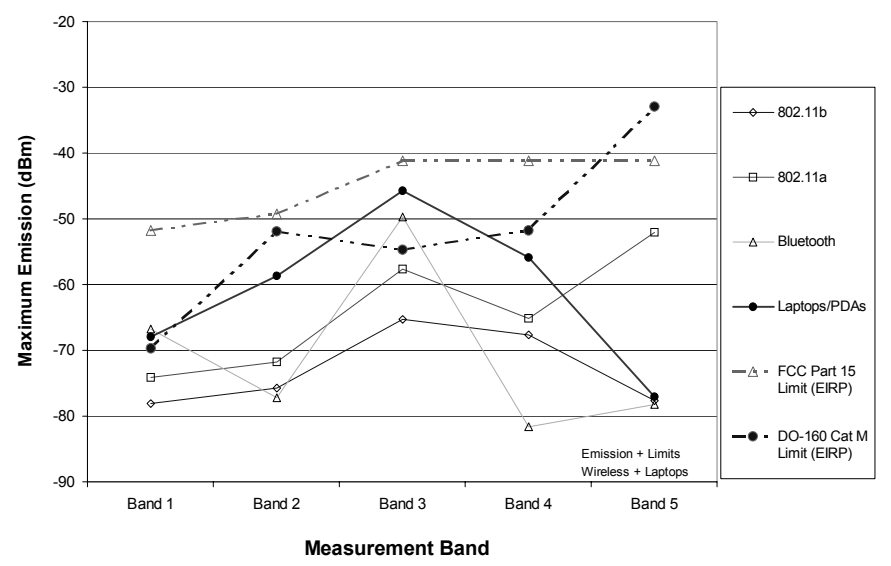

Figure 10. WLAN and Laptop Computer Emissions vs. Limits.

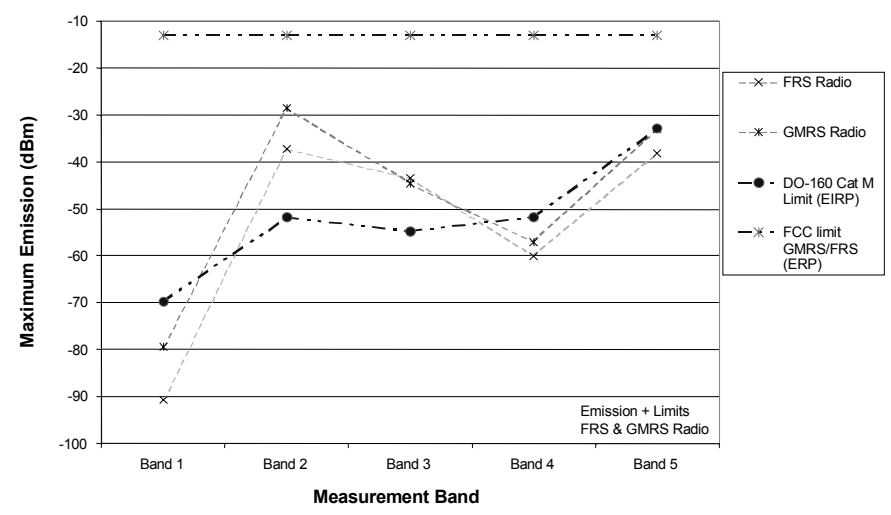

Figure 11. FRS/GMRS Radio Emission vs. FCC andRTCA/DO-160 Limits.

emissions from the common laptop computers and PDAs that are currently allowed during flights. The exception is the MLS band, which is not commonly used in the US.

2. Spurious emissions from the WLAN devices were lower than Federal Communications Commission (FCC) Part
15 limits, but were higher than aircraft RTCA/DO-160D Category M emission limits in the TCAS, and DME bands.

3. FRS/GMRS radio emissions were much higher than from the laptops/PDAs in the GS and MLS bands. In the GS band, emissions from GMRS/FRS radios exceeded the laptop/PDA maximum emission by as much as $30 \mathrm{~dB}$, and the aircraft emission limit RTCA/DO-160D Category M by as much as $23 \mathrm{~dB}$. In the MLS band, the maximum emissions from GMRS/FRS radios exceeded the laptop/PDA maximum emissions by at least $44 \mathrm{~dB}$, but were below the RTCA/DO160D Category $\mathrm{M}$ emission limit.

\section{CONCLUSIONS}

Spurious emissions in aircraft radio band from tested WLAN devices were not of greater threat than emissions from laptop computers and PDAs tested, with the exception of MLS band. However, emissions from FRS and GMRS radios were significantly higher than both the aircraft equipment emission limits and laptop computers' and PDAs emissions in two of the aircraft radio bands.

\section{REFERENCES}

[1] T. X. Nguyen, S. V . Koppen, J. J. Ely, R. A. Williams, L. J. Smith, and M. T. Salud, "Portable Wireless LAN Device and Two-Way Radio Threat Assessment for Aircraft Navigation Radios", NASA/TP-2003-212438, July 2003.

[2] RTCA DO-160D, Change No. 1, Section 21, "Environmental Conditions and Test Procedures for Airborne Equipment", Prepared by SC-135, December 14, 2000.

[3] 47CFR Ch. 1, Part 15.109, "Radiated Emission Limits", US Code of Federal Regulations, Federal Register dated December 19, 2001.

[4] 47CFR Ch. 1, Part 15.209, "Radiated Emission Limits; General Requirements", US Code of Federal Regulations, Federal Register dated December 19, 2001

[5] G. Koepke, D. Hill, and J. Ladbury, "Directivity of the Test Device in EMC Measurements", 2000 IEEE International Symposium on Electromagnetic Compatibility, Aug. 21-25, 2000.

[6] 47CFR Ch. 1, Part 95.635, "Personal Radio Service - Unwanted Radiation", US Code of Federal Regulations, Federal Register dated 10-01-98. 